\title{
Discovery of weak magnetic fields in early B-type stars
}

\author{
Huib F. Henrichs, Coralie Neiner, and Vincent C. Geers \\ Sterrenkundig Instituut Anton Pannekoek, Universiteit van Amsterdam, \\ Kruislaan 403, NL-1098 SJ, Nederland
}

\begin{abstract}
We summarize recent results of magnetic measurements of three bright early B-type stars, $\beta \mathrm{Cep}, \zeta \mathrm{Cas}$, and V2052 Oph, which were found to be oblique rotators with a weak magnetic dipole field with typical strength of a few hundred Gauss. From stellar wind studies we could derive their rotational periods very accurately, and match the stellar wind with the magnetic phase. From model atmosphere fits we derive the angles of rotational and magnetic axis. All three stars show some chemical abundance anomaly, presumably associated with the magnetic properties. The stars are also pulsating variables. This is of high asteroseismological interest, since these are the only early-type stars known with observationally determined rotation, pulsation and magnetic properties.
\end{abstract}

One of the major questions in early-type stars research is why their winds show cyclic behaviour on a rotational timescale, which is typically a few days. Two types of corotating surface phenomena, non-radial pulsations (or beating in case of multiple modes) and the presence of surface magnetic fields, have been proposed to cause the necessary azimuthal perturbation at the base of the wind. Observed timescales of non-radial pulsations are in all cases, however, far too short. Beating of multiple modes has been observed in one Be star, but the conjecture that in all other stars the present modes conspire to have their beating period match the rotational timescale is hard to defend. Our research has therefore concentrated on directly measuring magnetic fields in early OB stars, of which the strength is of order $100 \mathrm{G}$. So far no detection has been made for a 'normal' OB star with a cyclic wind, but we recently discovered that these weak fields at least exist in some early B-type stars, which we report here.

During more than 15 years we have observed specific wind behaviour in UV lines of a number of early B-type stars, of which no magnetic detection was known. The (presumably) rotation periods could be very accurately derived, which was essential to discover their (weak) surface fields. This clearly demonstrates that even these weak field strongly influence the wind behaviour.

Magnetic measurements were carried out with the Musicos spectropolarimeter attached to the $2 \mathrm{~m}$ Telescope at the Pic du Midi, France. We summarize in Table 1 the results for $\beta$ Cep (Donati et al. 2001; Henrichs et al. 2002), $\zeta$ Cas (Neiner et al. 2002b), and V2052 Oph (Neiner et al. 2002a). We refer to these papers for the technique used, and detailed discussions In all cases we observed the longitudinal component of the magnetic field, averaged over the stellar disk, which showed a sinusoidal behavior correlated with the stellar wind. From the amplitude of typically 50-100 G and a detailed model fit to optical spectral lines, 
Table 1. Summary of derived stellar parameters and magnetic properties.

\begin{tabular}{lcccccccccc}
\hline \hline star & $\begin{array}{c}\text { sp. } \\
\text { type }\end{array}$ & $V$ & $\begin{array}{c}M \\
\left(\mathrm{M}_{\odot}\right)\end{array}$ & $\begin{array}{c}R \\
\left(\mathrm{R}_{\odot}\right)\end{array}$ & $\begin{array}{c}T_{\text {eff }} \\
(\mathrm{K})\end{array}$ & $\begin{array}{c}v \sin i \\
\left(\mathrm{kms}^{-1}\right)\end{array}$ & $\begin{array}{c}P_{\text {rot }} \\
(\mathrm{d})\end{array}$ & $\begin{array}{c}i \\
\left({ }^{\circ}\right)\end{array}$ & $\begin{array}{c}\beta \\
\left({ }^{\circ}\right)\end{array}$ & $\begin{array}{c}B_{\text {pol }} \\
(\mathrm{G})\end{array}$ \\
\hline$\beta$ Cep & B1 IV & 3.2 & 12 & 6.4 & 26000 & 27 & 12.00106 & 60 & 85 & 360 \\
$\zeta$ Cas & B2 IV & 3.7 & 8 & 5.9 & 20400 & 17 & 5.37045 & 18 & 80 & 340 \\
V2052 Oph B1 V & 5.8 & 10 & 4.1 & 25200 & 60 & 3.63883 & 71 & 60 & 250 \\
\hline \hline
\end{tabular}

we could derive the polar field of the star, which is between 200 and $400 \mathrm{G}$.

Abundance anomalies. Gies \& Lambert (1992) noted that $\beta$ Cep and $\zeta$ Cas are $\mathrm{N}$-enriched stars. We confirm this for $\zeta$ Cas, and found that V2052 Oph is a He-strong star. Such chemical peculiarities are expected in a magnetic environment. Our study confirms that even such weak fields have a strong impact on the surface properties.

Discussion and future work. X-ray emission observed in $\beta$ Cep has been shown by Donati et al. (2001) to be consistent with the model by Babel \& Montmerle (1997) in which the wind flow is magnetically confined. A significant rotationally modulated X-ray flux is predicted for all three stars. Much progress in modeling is expected from new dynamical simulations of magnetically channeled stellar winds (ud-Doula \& Owocki 2002; these Proceedings). New imminent instrumentation (like ESPADONS and NARVAL with at least ten times more magnetic sensitivity) will undoubtedly greatly help to improve these results, and will allow to discover more of stars in this class, and hopefully in O-type stars as well.

Towards Asteroseismology. All three studied stars are pulsating variables: $\beta \mathrm{Cep}$, and V2052 Oph are $\beta$ Cephei stars, whereas we identified $\zeta$ Cas as a Slowly Pulsating B-type star. This shared property opens exciting asteroseismological applications: since the rotation, pulsation modes and magnetic field are known, no free parameter is left, and the stellar parameters and evolutionary stage can be strongly constrained. This principle was shown to work for $\beta$ Cep by Shibahashi \& Aerts (2000), although their analysis need significant improvement.

Acknowledgments. We gratefully thank the various observers who contributed to this program: J. Nichols, J. de Jong, J.-F. Donati, E. Verdugo, A. Talavera, A. Tijani and K. Wiersema. We also thank them and Danny Lennon for stimulating discussions.

\section{References}

Babel, J., Montmerle, T. 1997, A\&A 323, 121

Donati, J.-F., Wade, G., Babel, J., Henrichs, H.F., et al. 2001, MNRAS 326, 1165

Gies, D.R., Lambert, D.L. 1992, ApJ 387, 673

Henrichs, H.F., de Jong., J., Donati, J-F., Verdugo, E., et al. 2002, in preparation Neiner, C., Henrichs, H.F., Floquet, M., Frémat, Y., et al. 2002a, A\&A submitted Neiner, C., Geers, V.C., Henrichs, H.F., Floquet, M., et al. 2002b, A\&A in press Shibahashi, H., Aerts, C. 2000, ApJ (Letters) 531, L143

ud-Doula, A., Owocki, S.A. 2002, ApJ 576, 413 